\title{
Understanding microstructural deformation of apple tissue from 4D micro-CT imaging
}

\author{
Zi Wang ${ }^{1}$, Seppe Rogge ${ }^{1}$, Metadel Abera ${ }^{1}$, Mattias van Dael ${ }^{1}$, Vincent Van Nieuwenhove ${ }^{3}$, Pieter \\ Verboven ${ }^{1}$, Jan Sijbers ${ }^{3}$, Bart Nicolai ${ }^{1,2 *}$ \\ ${ }^{1}$ MeBioS, Department of Biosystems, KU Leuven, Willem de Croylaan 42, 3001 Leuven, Belgium \\ 2Flanders Centre of Postharvest Technology, Willem de Croylaan 42, 3001 Leuven, Belgium \\ 3University of Antwerp, iMinds-Vision Lab, Universiteitsplein 1, Antwerp, Belgium \\ *Corresponding author, email: bart.nicolai@biw.kuleuven.be
}

Keywords: Apples, micro-CT, dehydration, deformation, microstructure, 4D

\section{INTRODUCTION}

The production of Apples and derivative products are economically important for many EU countries (FAOSTAT, 2016). Since fruit quality effectively determine their sale price, preservation of fruit quality is vital to maintaining their market value following harvest. Currently, Apples are stored in warehouses with controlled temperature, respiratory gas concentration and humidity levels. Even so, losses from storage disorders have been observed in multiple commercial cultivars under long term storage (Elgar et al., 1999; Ho et al., 2010; Kittemann et al., 2015). Further optimization is therefore necessary to reduce losses during storage. However, such improvements are exceptionally difficult to make as multiple environmental parameters must be properly balanced. Principles from thermal dynamics, fluid dynamics, gas exchange, and water transport must be applied accordingly to achieve this. Given the complexity of these studies and resource limitations, computational models are utilized in in silico studies instead of scaled experiments.

To study water transport and tissue dehydration of apple tissue, currently utilized computational models are based on an effective diffusion approach, where average water content can be predicted utilizing the apparent model parameters that are experimentally derived to fit a diffusion equation (Aregawi et al., 2013). However, current models operate under the assumption that the tissue is homogenous in nature, whereas it actually consists of turgid cells of varying shapes and sizes along with an embedded network of intercellular air spaces (Herremans et al., 2015). Thus, the approach of utilizing empirical data to calibrate and fit the dehydration process of apples onto existing diffusion type models raises two fundamental questions. First, can the dehydration and deformation of apple tissue truly be described as a diffusion process? Second, can the model be improved if the process was described on a cellular scale? To answer these questions, cellular scale models are currently being developed to understand the process of water transport and the associated mechanics. Internal unpublished results currently incorporate the loss of turgor during the dehydration process, thus the inclusion of deformation takes a fundamentally different approach than the effective diffusion assumption. However, to properly construct the new models, experimental data in $4 \mathrm{D}(3 \mathrm{D}+$ time $)$ is necessary for calibration and validation.

To obtain 4D data on a highly dynamic process such as dehydration and deformation, significant challenges exist. Micro-CT instruments that can provide microstructure data of fruit cortex tissue typically require long scan times (Mendoza et al., 2007), which inevitably results in very low temporal resolution. Moreover, as dehydration and deformation by definition causes movement, image artifacts such as motion blurring and image doubling are inevitable in the reconstructed images from these long scans, which makes the downstream analysis of the data 
exceptionally difficult if not impossible. Currently, there are two separate approaches to counteract the motion artifacts. First, a fast scanner with a modified scanning sequence could be utilized to reduce the scanning duration and limit the degree of movement of the sample, however this is typically done at the cost of higher noise in the final reconstructed image. Second, dynamic reconstruction techniques (Van Eyndhoven et al., 2015) based on the original iterative reconstruction techniques (Gilbert, 1972) can be used in combination with the fast scans to significantly reduce noise, but at the cost of increased computational time and hardware requirements. In this paper, we examine the feasibility of using a modified fast scan approach to obtain 4D data on apple tissue dehydration and deformation.

\section{MATERIALS AND METHODS}

\section{Samples and preparation}

Apples of the "Kanzi" cultivar (Malus $\times$ domestica Borkh) from the 2015 harvest season were utilized for this study. The apples were stored at $4^{\circ} \mathrm{C}$ until needed for experimentation. An apple cortex tissue sample approximately $4 \mathrm{~mm} \times 3.2 \mathrm{~mm} \times 4.5 \mathrm{~mm}$ (L x W x H) was extracted using an ultra-thin razor blade $(\sim 0.125 \mathrm{~mm}$ thickness $)$ to reduce cutting induced damage. Excess water from cutting the sample was wicked away with Kimwipes prior to mounting the sample onto a plastic sample holder with double sided tape.

\section{Scanning parameters and chamber conditions}

The extracted cortex sample was scanned with a GE Phoenix Nanotom microCT system (GE Sensing \& Inspection Technologies GmbH, Wunstorf, Germany) with $55 \mathrm{kV}$ and $180 \mu \mathrm{A}$ applied to the X-Ray tube. 600 projection images were captured per dataset with a stepping angle of $0.6^{\circ}$ over a $360^{\circ}$ rotation. Detector exposure time was set to $500 \mathrm{~ms}$, which limited the total acquisition time per scan to $5 \mathrm{~min}$. Scans with a voxel size of 3.0 microns were taken at $10 \mathrm{~min}$ intervals until $240 \mathrm{~min}$ have elapsed resulting in 25 image sets. The scanning chamber was controlled at approximately $25^{\circ} \mathrm{C}$ with a relative humidity of $50 \%$.

\section{Reconstruction and image processing}

Image reconstruction was done via the Octopus Reconstruction suite (Inside Matters, Gent, Belgium) with the embedded filtered back projection (FBP) algorithm along with a Fourier filter applied to the sinograms to remove excessive noise. The final reconstructed images were downscaled to 8-bit to reduce computational requirements and a fixed greyscale range was applied to ensure consistency of the 25 datasets. An automated data processing workflow similar to (Herremans et al., 2015) was utilized to obtain analyzable binary images for further analysis.

\section{Data analysis}

Binary images were quantified using Avizo (FEI, Bordeaux, France) and cell volume information was extracted along with the centroid of individual segmented cells. Cells were subsequently divided into arbitrarily defined inner, middle and outer layers via MATLAB (MathWorks, Natick, USA) and cell volume information was further binned by its 3 dimensional positioning within the defined layers and exported for further analysis. Cell volume distribution was determined and plotted via Prism (GraphPad Software, La Jolla, USA).

\section{RESULTS AND DISCUSSION}

As previously mentioned, in order to properly derive models on the dehydration and deformation process of apple cortex tissue, relevant biological data must be obtained to calibrate and validate these models during its construction. 


\section{D data of apple cortex tissue during dehydration}

By utilizing a modified scanning protocol of a commercially available fast X-Ray microCT scanner, we have demonstrated for the first time the viability of utilizing X-Ray microCT to capture fruit cortex microstructure data in 4D with sufficient spatial and temporal resolution. Rapid dehydration and deformation can be observed visually within the time series (Figure 1), where cell sizes decreases considerably during the first two hours of the experiment.

Initial analysis of the sub regions defined in the sample data set (Figure 2) indicate a significant decrease $(\mathrm{P}<0.05)$ in cell volume in both outer and middle layer by $30 \mathrm{~min}$. Whereas the inner layer was shielded from such effect until approximately 90 min into the experiment (Figure 3). Given that these changes are visually observable within the reconstructed images, such results are not surprising. However, the analysis presented in this study is incomplete as the difficulty of analyzing dynamic data is challenging and significant improvements must be made to the methods in which the data is analyzed. For instance, having a fixed bounding box and predefined zones may work for dynamic samples with minor deformation, but in severe cases such as the dataset presented in this study, subsequent analysis based on fixed bounding boxes may introduce artifacts to the analysis results. Ideally, parameters such as porosity, cell size/ equivalent spherical diameter, convexity should be analyzed, but the minimum representative volume for a dynamic sample is currently unknown, which compounds the difficulty of the subsequent analysis. Moreover, as deformation occurs within the sample, the ability to track the movement as well as geometric characteristics of individual cells will yield interesting data on the dehydration and deformation process. However, given the sheer number of cells and the complexity of the dynamic dataset, tracking individual objects via spatial correlation is also a significant challenge.

\section{Cortex tissue dehydration process}

From the observations it can be hypothesized that the dehydration process is mainly characterized by water loss from cells by permeation and diffusion through the membrane and cell walls to the surrounding air spaces resulting in cell shrinkage affecting mechanical deformation of the entire tissue sample due to changes in the stress equilibrium between cells. Large changes of local pore size are also seen. As a result the amount of water per unit volume in each layer is changing. When observed on a larger scale this could be seen as a developing volumetric water concentration gradient that could be interpreted as an apparent diffusion process, as it is commonly described. Further exploration of the changes may require microscale modelling and combination with imaging approaches to visualize water content (such as MRI or neutron imaging). In further steps, the water transport in entire fruit across different tissues, cuticle and wax layers should be investigated.

\section{Refinements to methodology}

Although the current protocol can provide 4D data sets with good spatial and temporal resolution, some movement artifacts such as motion blurring and image doubling can still be observed. Further reduction in acquisition time is therefore necessary to counteract the motion artifacts. However, given that detector exposure time cannot be further reduced without a severe penalty on the signal to noise ratio, only remaining site of intervention is increasing the stepping angel and thus reducing the number of captured projection images. Given that the FBP algorithm is analytical in nature and require a large number of projections for high resolution scans, a reduction in the number of projection images inevitably suffers from interpolation effects and high noise (Penczek, 2010). Nevertheless, with the incorporation of iterative reconstruction methods such as simultaneous iterative reconstruction technique (SIRT) into the modified scanning protocol to further reduce scan time and thus motion artifacts albeit at higher computational requirements. Preliminary internal testing (data not shown) indicate that scan time can be reduced to as little as $90 \mathrm{~s}$ without discernible losses in reconstructed image quality. 
Thus, the combined protocol appears to have the benefit of being able to reduce motion artifacts while simultaneously increasing the temporal resolution of the $4 \mathrm{D}-\mu \mathrm{CT}$ protocol.

\section{CONCLUSION}

4D microstructure data of dehydrating apple cortex tissue can be provided by a commercially available fast X-Ray microCT scanner with a modified scanning protocol. Moreover, initial analysis of the sample dataset indicate that the mechanics of dehydration and deformation at the cellular scale is likely not a diffusion process. New models of apple cortex dehydration must therefore be constructed based on the new $4 \mathrm{D}-\mu \mathrm{CT}$ data to more accurately simulate water transport in apple cortex tissue.

\section{ACKNOWLEDGEMENTS}

The authors acknowledge financial support of the Research Fund - Flanders (FWO SBO project 120033).

\section{Literature cited}

Aregawi, W., Defraeye, T., Saneinejad, S., Vontobel, P., Lehmann, E., Carmeliet, J., Derome, D., Verboven, P., and Nicolai, B. (2013). Dehydration of apple tissue: Intercomparison of neutron tomography with numerical modelling. Int. J. Heat Mass Transf. 67, 173-182.

Elgar, J.H., Burmeister, D.M., and Watkins, C.B. (1999). Harvest date and crop load effects on a carbon dioxide-related storage injury of "Braeburn” apple. HortScience 34, 305-309.

Van Eyndhoven, G., Batenburg, K.J., Kazantsev, D., Van Nieuwenhove, V., Lee, P.D., Dobson, K.J., and Sijbers, J. (2015). An Iterative CT Reconstruction Algorithm for Fast Fluid Flow Imaging. IEEE Trans. Image Process. 24, 4446-4458.

FAOSTAT (2016). http://faostat3.fao.org.

Gilbert, P. (1972). Iterative methods for the three-dimensional reconstruction of an object from projections. J. Theor. Biol. 36, 105-117.

Herremans, E., Verboven, P., Verlinden, B.E., Cantre, D., Abera, M., Wevers, M., and Nicolaï, B.M. (2015). Automatic analysis of the 3-D microstructure of fruit parenchyma tissue using X-ray micro-CT explains differences in aeration. BMC Plant Biol. 15, 264.

Ho, Q.T., Verboven, P., Verlinden, B.E., Schenk, A., Delele, M.A., Rolletschek, H., Vercammen, J., and Nicolai, B.M. (2010). Genotype effects on internal gas gradients in apple fruit. J. Exp. Bot. 61, 2745-2755.

Kittemann, D., Neuwald, D.A., and Streif, J. (2015). Internal browning in "kanzi" apples - reasons and possibilities to reduce the disorder. Acta Hortic. 409-414.

Mendoza, F., Verboven, P., Mebatsion, H.K., Kerckhofs, G., Wevers, M., and Nicolaï, B. (2007). Three-dimensional pore space quantification of apple tissue using X-ray computed microtomography. Planta 226, 559-570.

Penczek, P.A. (2010). Fundamentals of Three-Dimensional Reconstruction from Projections. In Methods in Enzymology, (Elsevier Inc.), pp. 1-33. 


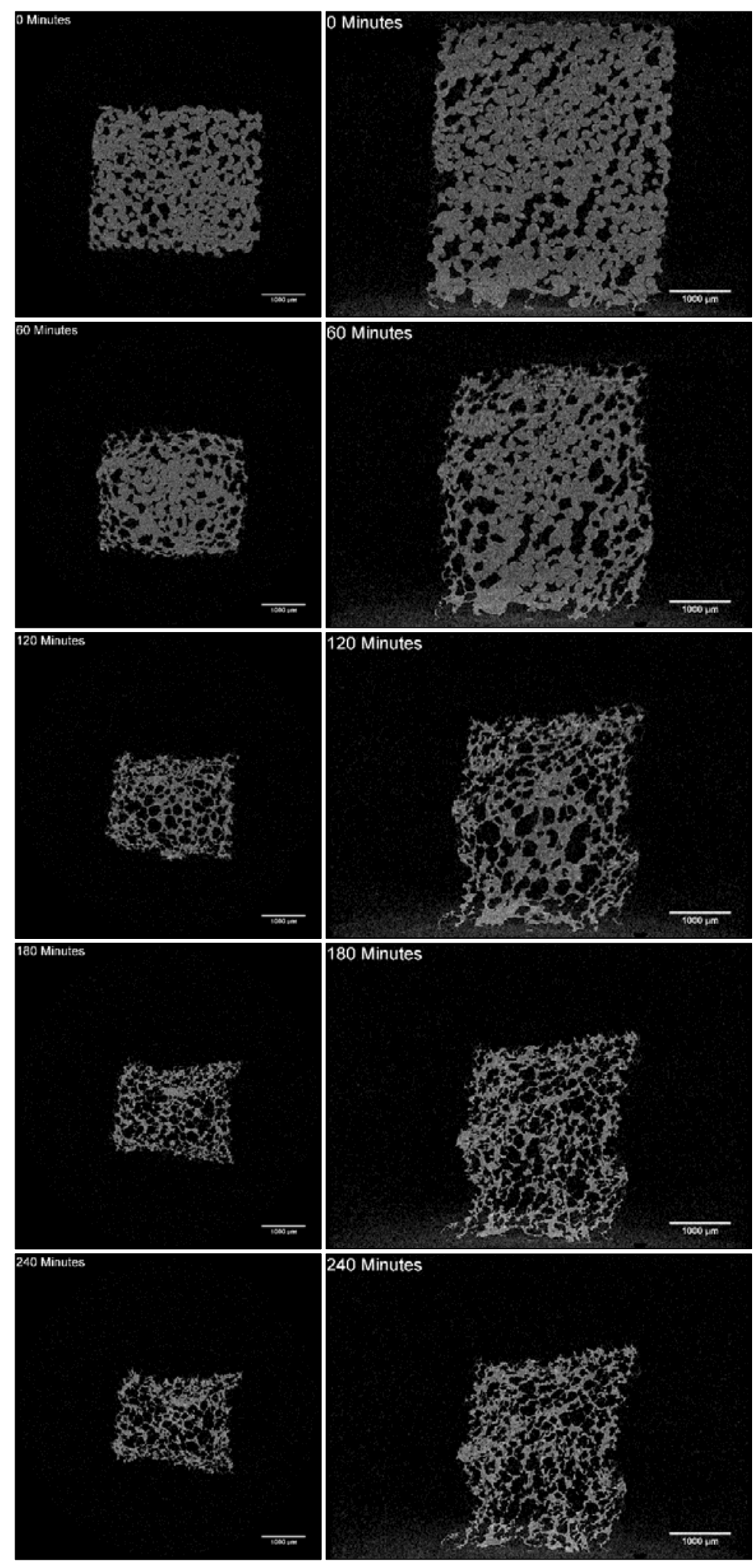

Figure 1. XY (left) and XZ (right) sections of apple microstructure over the dehydration timeframe. 

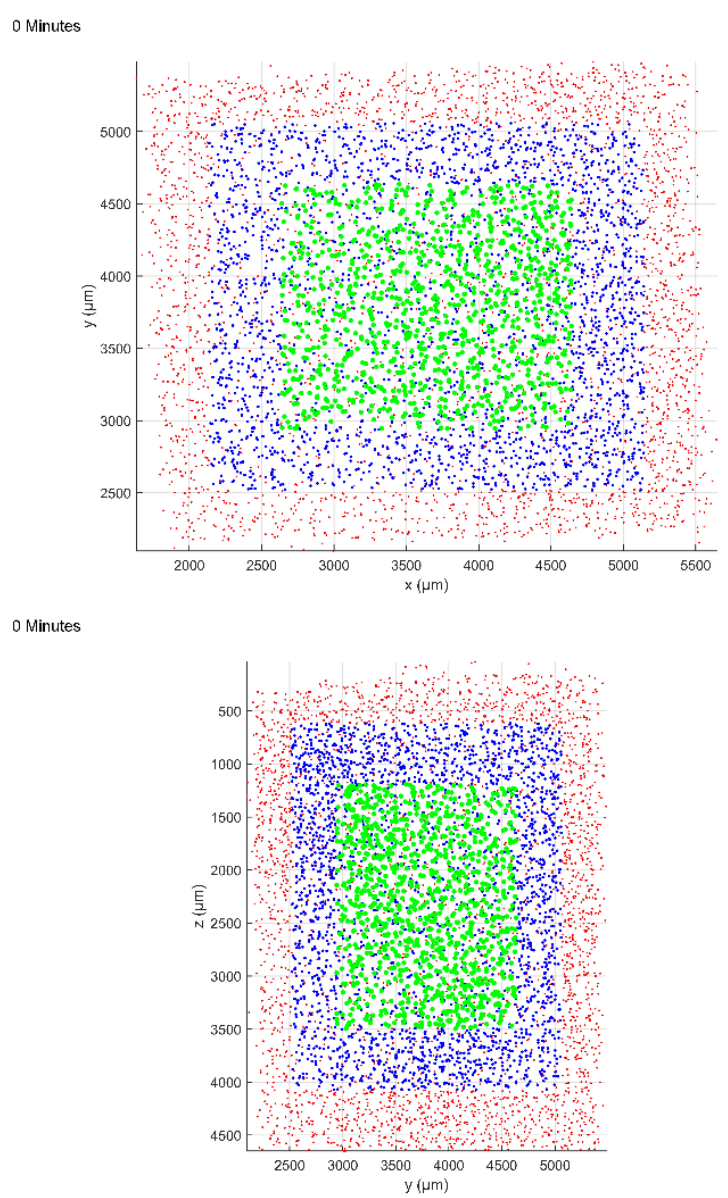

Figure 2. XY (left) and YZ (right) centroid plots of the cells in the outer, middle and inner layers defined in the initial scan in red, blue and green respectively. 
Cell size distribution in the outer layer

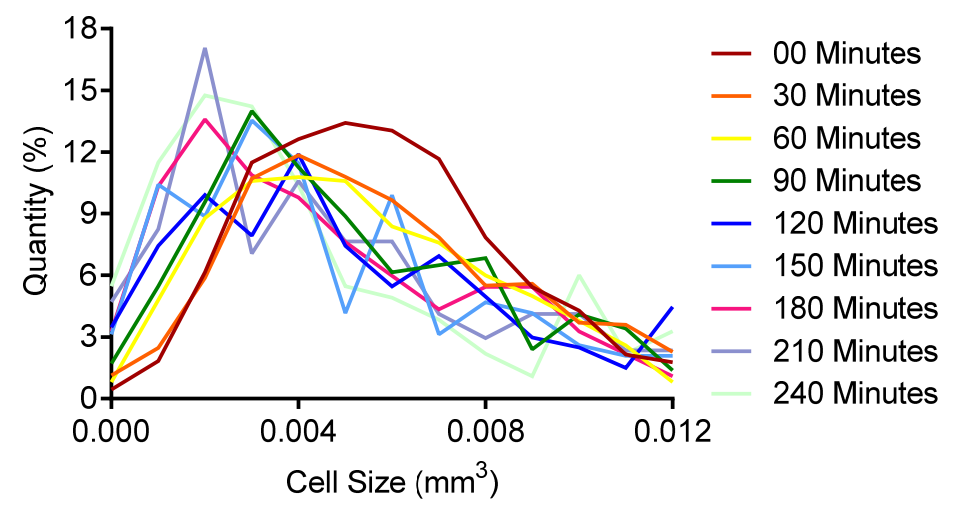

Cell size distribution in the middle layer

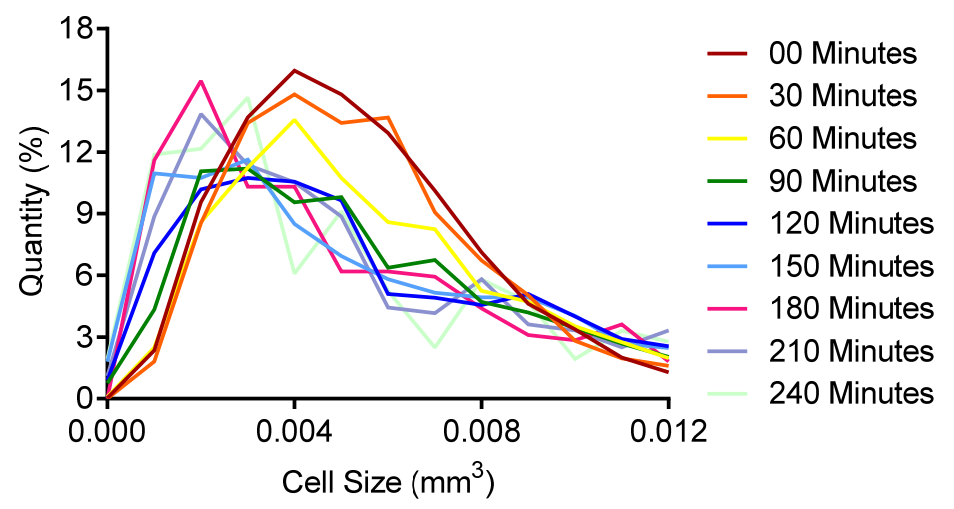

Cell size distribution in the inner layer

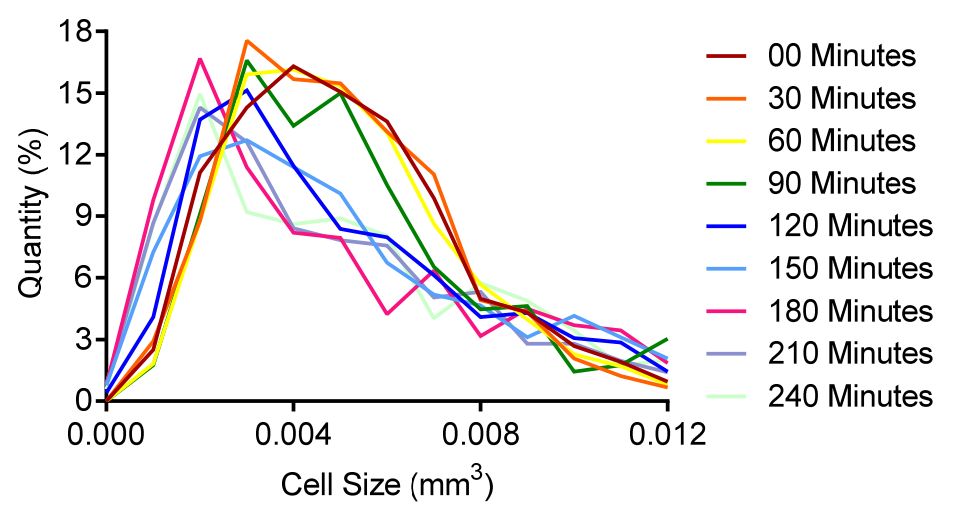

Figure 3. Cell sizes and their distribution at 30 min intervals over the experimental dehydration period. 